\title{
tic\&société
}

Vol. 11, $\mathbf{N}^{\circ} 1$ | 2ème semestre 2017

L'éducation critique aux médias à l'épreuve du numérique

\section{Un modèle pour repenser l'éducation critique aux médias à l'ère du numérique}

Pierre FASTREZ et Thibault PHILIPPETTE

\section{OpenEdition}

\section{Journals}

Édition électronique

URL : http://journals.openedition.org/ticetsociete/2266

DOI : 10.4000/ticetsociete.2266

Éditeur

Association ARTIC

Édition imprimée

Pagination : 85-110

\section{Référence électronique}

Pierre FASTREZ et Thibault PHILIPPETTE, « Un modèle pour repenser l'éducation critique aux médias

à l'ère du numérique », tic\&société [En ligne], Vol. 11, N 1 | 2ème semestre 2017, mis en ligne le 01 septembre 2017, consulté le 10 décembre 2020. URL : http://journals.openedition.org/ticetsociete/ 2266 ; DOI : https://doi.org/10.4000/ticetsociete.2266 
tic\&société - 11(1), 2017

\title{
Un modèle pour repenser l'éducation critique aux médias à l'ère du numérique
}

\author{
Pierre FASTREZ
}

Pierre Fastrez est chercheur qualifié du Fonds national de la recherche scientifique (F.R.S.-FNRS) belge et professeur à l'École de communication (COMU) et au Centre de recherche en communication (RECOM) de l'Université catholique de Louvain (Belgique). Ses recherches portent sur l'influence des technologies de l'information et de la communication sur les activités cognitives qu'elles soutiennent et sur les compétences de littératie médiatique. Dans ce second domaine de recherche, il s'intéresse particulièrement à la définition conceptuelle de la littératie médiatique et au développement d'outils d'évaluation des niveaux de littératie médiatique.

pierre.fastrez@uclouvain.be

\section{Thibault PHILIPPETTE}

Thibault Philippette est professeur associé à l'École de communication de l'Université catholique de Louvain, où il donne un cours en master intitulé "Littératie médiatique ». II est également membre du Groupe de recherche en médiation des savoirs (GReMS) et du Laboratoire Jeux et Mondes virtuels (LabJMV). Ses domaines de recherche portent sur l'appropriation sociale des technologies, les compétences numériques et la littératie médiatique, l'étude des activités de jeu (play studies) et la place du ludique dans les formes médiatiques contemporaines (ludologie médiatique).

thibault.philippette@uclouvain.be 


\section{Un modèle pour repenser l'éducation critique aux médias à l'ère du numérique}

Résumé : L'éducation aux médias a depuis toujours placé au centre de son projet le développement d'une pensée critique face aux médias. Dans cet article, nous discutons dans un premier temps de la définition du qualificatif "critique ", appliqué à différents objets (posture, pédagogie, situation et action). Nous présentons ensuite un modèle de la littératie médiatique sur la base duquel nous explorons l'étendue possible d'une approche critique des compétences médiatiques dans le contexte des médias numériques. Ce faisant, nous mettons au jour une conception large de cette approche, articulant les perspectives technique, informationnelle et sociale sur les médias, tant en contexte de réception que de production, dépassant ainsi l'approche traditionnelle de l'éducation aux médias, qui cantonne la pensée critique à des compétences de lecture socio-informationnelle.

Mots-clés : littératie médiatique, pensée critique, compétence, sociomatique.

Abstract: Media education has always placed the development of critical thinking about media at the core of its project. In this article, we first discuss the definition of the adjective "critical" when applied to different objects (posture, pedagogy, situation and action). We then present a model of media literacy with which we explore the possible scope of a critical approach to media competencies in the context of digital media. In doing so, we propose a broader conception that connects technical, information and social perspectives on media, both in the context of their reception and their production, thereby extending beyond the traditional approach to media education, which restricts critical thinking to socio-informational reading skills.

Keywords: media literacy, critical thinking, competency, sociomatics. 
Un modèle pour repenser l'éducation critique aux médias à l'ère du numérique

Resumen: La educación sobre los medios siempre ha colocado en el centro de su proyecto el desarrollo de un pensamiento crítico sobre los medios. En este artículo, se discute, en primer lugar, la definición del adjetivo "crítico", aplicado a diferentes objetos (postura, pedagogía, situación y acción). Después, se propone un modelo de alfabetización mediática que servirá de base para explorar la posible extensión de un enfoque crítico de las competencias mediáticas en el contexto de los medios digitales. De esta forma, se ofrece una concepción amplia de este enfoque, que articula las perspectivas técnicas, informacionales y sociales de los medios, tanto en el contexto de recepción como en el de producción. De este modo, la propuesta trasciende el enfoque tradicional de la educación sobre medios, que restringe la reflexión crítica a las competencias de lectura socioinformacional.

Palabras claves: alfabetización mediática, pensamiento crítico, competencia, sociomática. 


\section{L'éducation critique aux médias}

La déclaration de Grünwald de I'UNESCO (1982) souligne que les systèmes politiques et éducatifs ont l'obligation de promouvoir auprès de leurs citoyens une "compréhension critique " des phénomènes de communication que sont les médias. Cette volonté politique de développer une pensée critique vis-à-vis des médias est donc inscrite dès le départ dans le projet de l'éducation aux médias. Ceci étant dit, la question est de savoir ce que sous-tend cette idée de compréhension " critique », pour ensuite pouvoir envisager les manières de la promouvoir.

Cet article s'intéressera, dans un premier temps, à ce que critique signifie dans le contexte de l'éducation aux médias. Nous ne prétendons pas épuiser le sujet, qui a déjà fait couler beaucoup d'encre (p. ex. Alvermann et Hagood, 2000 ; Kellner et Share, 2005), mais bien de montrer le caractère polysémique de ce qualificatif, suivant les champs de recherche qui le mobilisent et selon l'objet sur lequel il porte.

Dans un deuxième temps, nous présenterons un modèle de littératie médiatique ${ }^{1}$ développé au sein de notre groupe de recherche (Fastrez, 2010; Fastrez et De Smedt, 2012). Celui-ci nous permettra, dans un troisième temps, et à travers plusieurs illustrations, d'envisager comment développer une " éducation critique aux médias ", incluant non seulement les capacités de lecture et de compréhension critique, mais également les activités de conception et de création qui se développent avec l'évolution des médias numériques ${ }^{2}$.

\subsection{Qu'est-ce qui est " critique " à l'ère du numérique?}

Le développement de capacités d'analyse et de jugement critiques est au cœur des propos de plusieurs théoriciens et praticiens en éducation aux médias (p. ex. Bazalgette, 1992 ;

${ }_{2}^{1}$ Ce concept sera détaillé un peu plus loin dans l'article.

2 Suivant Anderson (1988), qui définit un média comme « une activité humaine distincte qui organise la réalité en textes lisibles en vue de l'action » (p. 11), nous utilisons l'expression générique médias numériques, ici et ultérieurement, pour traiter de manière indifférenciée tous les médias dont le substrat technique est informatique, et donc numérique. Dans cette perspective, nous considérons ce qu'il est convenu d'appeler les technologies de l'information et de la communication (TIC) comme le substrat technique des médias (numériques ou non). 
Un modèle pour repenser l'éducation critique aux médias à l'ère du numérique

Buckingham, 1998 ; Hobbs, 1998 ; Livingstone, 2003). Traitant des médias de masse, ils constatent que l'éducation aux médias a pour fonction de développer l'esprit critique, ou plus directement ce que Masterman appelle l'autonomie critique chez les élèves, c'est-à-dire une confiance en soi et une maturité critique suffisantes pour pouvoir appliquer un jugement sur les textes médiatiques rencontrés ultérieurement (Masterman, 1990). La dimension «critique » en éducation aux médias renvoie à une posture "critique " transférable d'un contexte médiatique à un autre. Ce qui définit cette posture dépend néanmoins des perspectives et des cadres théoriques mobilisés.

\subsubsection{Adopter une posture critique}

Une première approche qui nous semble intéressante à ce propos vient de l'analyse faite par De Munck (2011) de la sociologie critique. Les cadres théoriques mobilisés sont différents, mais les réflexions apportées sont toutefois éclairantes pour penser ce que signifie adopter une posture critique. L'auteur souligne que si l'on peut attribuer trois tâches à la sociologie en général (décrire le réel social, identifier les mal-fonctionnements dans la société et intervenir dans la réalité sociale), le propre de la sociologie critique est de vouloir articuler ces trois missions (De Munck, 2011). II pointe cependant deux risques à cela : celui d'être soit sur-inclusif, soit sous-inclusif. Le cas d'une posture sous-inclusive consisterait à ériger une tradition de pensée critique, souvent limitée à un auteur majeur (Bourdieu, Foucault, Marx, Habermas, etc.), en la transformant en doctrine à la base de toute critique. Dans le champ de l'éducation (aux médias), on peut également relever des textes faisant référence à un auteur unique pour repenser de manière critique l'école ou ses pédagogies ( $p$. ex. Andureau, 2003 ; Kline 2016). Le risque de sur-inclusivité, quant à lui, consisterait à étendre la critique de sorte à la faire équivaloir à toute forme de rationalité ou de réflexivité (De Munck, 2011). Pour De Munck, il faut davantage concevoir la critique en intention, sur le plan formel, comme recherche volontaire d'articulation entre théories explicatives, évaluation normative et intervention efficace avec les acteurs sociaux.

Si la réflexion menée par De Munck traite de la posture externe et critique du chercheur en sociologie, elle permet une 


\section{Pierre FASTREZ et Thibault PHILIPPETTE}

première réflexion sur ce que serait la posture critique adoptée par tout individu vis-à-vis des médias, voie médiane entre l'adoption d'une perspective analytique unique et la réflexivité au sens le plus générique.

Selon Feuerstein (1999), le rôle assigné à la pensée critique dans les curricula scolaires s'est développé depuis les années 1980, notamment en relation avec la littératie médiatique. L'auteure lie tout d'abord cette pensée critique à la métacognition, en tant que capacité réflexive sur ses propres processus de pensée. Piette (1996) souligne également le rôle fondamental de cette aptitude réflexive dans la construction d'une pensée critique. Ensuite, Feuerstein relève que cette pensée critique permet l'articulation de perspectives multiples face à des problèmes multidimensionnels et l'examen des systèmes de croyances sous-jacents à chaque perspective envisagée. Elle rejoint à ce titre les travaux de Paul (2012), qui souligne cette nécessité, pour pouvoir appréhender des problèmes aux multiples logiques, de mettre en œuvre une forme de pensée dialogique. Celle-ci doit permettre de s'abstraire des systèmes de croyance "egocentriques " et irrationnels que tout individu adopte suivant ses propres centres d'intérêt et systèmes d'appartenance: ethnique, religieux, socio-culturel, etc. (Paul, 2012.)

On le comprend, la pensée critique tient à une dynamique de décentration et d'articulation de différents points de vue sur un sujet complexe.

\subsubsection{La littératie médiatique critique}

Le concept de littératie médiatique, dans son acception générale, est un néologisme créé à partir de la traduction française de media literacy. II recouvre l' " ensemble des compétences caractérisant l'individu capable d'évoluer de façon critique et créative, autonome et socialisé dans l'environnement médiatique contemporain " (Fastrez, 2010, p. 36). II permet de distinguer d'une part la littératie médiatique, soit les compétences mobilisant les connaissances, les savoir-être et les savoir-faire relatifs aux médias, et l'éducation aux médias d'autre part, soit le processus éducatif visant le développement de ces compétences. 
Un modèle pour repenser l'éducation critique aux médias à l'ère du numérique

Aux États-Unis, Kellner et Share (2007) voient la critical media literacy, ou littératie médiatique critique, comme une extension de la media literacy. Les auteurs relèvent, tout comme d'autres, les filiations de ce courant avec les études féministes ou de genre, ainsi qu'avec les media and cultural studies (Alvermann et Hagood, 2000 ; Durham et Kellner, 2001; Kellner et Share, 2005). La littératie médiatique traditionnelle peut être selon eux considérée comme l'une des trois approches en éducation aux médias, aux côtés des approches protectionnistes et des approches plus esthétiques ou artistiques (Kellner et Share, 2007). La littératie médiatique critique, quant à elle, articulerait des perspectives issues de ces trois approches, tels que l'analyse et la déconstruction des formes de représentation (p. ex. Gainer, 2010 ; O’Byrne, 2012 ; Scharrer, 2005), l'étude du contexte social et des systèmes de domination idéologique et/ou politique ( $p$. ex. Papola-Ellis et Eberly, 2015 ; Smith 2013), ou encore l'examen des formes médiatiques alternatives aux productions dominantes (Kellner et Share, 2007).

La littératie médiatique critique est souvent accolée à des formes de pédagogies inspirées notamment de Paolo Freire et de Henry Giroux, qu'elles soient qualifiées d'éducations " critiques » (Gur-Ze'ev, 2000), « radicales » (Tinson et McBride, 2013) ou "transformatives" (Tisdell, 2008). Ces pédagogies cherchent à créer entre les apprenants un dialogue visant son internalisation sous la forme d'une posture critique tenant compte de multiples points de vue des sous-cultures participant d'un même environnement médiatique (Kellner et Share, 2007 ; Paul, 2012).

\subsection{Posture, situations et capacités d'action critiques}

Nous l'avons vu, le qualificatif de "critique " renvoie en première lecture à l'adoption d'une pensée critique, correspondant à un mouvement réflexif permettant l'expression d'un jugement de valeurs, souvent associé, dans la littérature, à des principes démocratiques et de justice sociale (Giroux, 1983 ; Kellner et Share, 2007). Cependant, il ne doit pas être pour autant confondu avec toute forme de rationalité objective, présupposant qu'il est possible de juger d'une situation 
médiatique donnée à partir d'une posture définie a priori. II nous semble nécessaire, afin de rendre effective une posture critique, et d'éviter les phénomènes de sur- ou de sousinclusion relevés précédemment, de tenir compte d'autres paramètres. Nous en identifions au moins deux.

D'une part, une posture critique s'adopte en regard d'une situation critique, c'est-à-dire conçue comme le lieu d'un potentiel mal-fonctionnement social (cf. supra). Comme le relèvent Kellner et Share (2007), cela renvoie aux politiques de représentation (par exemple aux idéologies, aux omissions, aux rapports destinateur-destinataire) et à la perception d'un déséquilibre de position au sein d'une culture médiatique donnée. Dans le cas des médias contemporains, cela touche par exemple la perception des vulnérabilités par rapport aux systèmes numériques d'inclusion/exclusion (Brotcorne, et al., 2010). Être capable de percevoir et de problématiser les éléments critiques d'une situation médiatique nécessite d'avoir un ensemble riche de clés de lecture.

D'autre part, une posture critique doit soutenir des capacités d'action critique: à l'instar de la sociologie critique (cf. 1.1.2), elle doit donner lieu à une intervention transformative et plus démocratique de la situation médiatique. Si on pense une éducation critique aux médias comme une discipline cherchant à développer chez les apprenants une posture critique articulant par exemple la mobilisation de multiples cadres analytiques portant sur les médias (sémiotique, pragmatique, sociologie des usages, etc.) avec des capacités de jugement tenant compte des différents points de vue culturels des acteurs et des tensions entre ceux-ci, il faut également qu'elle puisse développer les capacités et les moyens d'une action critique sur cet environnement médiatique.

Dans le point suivant, nous présentons brièvement un modèle d'analyse de la littératie médiatique dont nous nous servirons ensuite pour situer la posture critique comme articulation de perspectives multiples sur les objets médiatiques et penser une éducation critique aux médias à l'ère du numérique. 
Un modèle pour repenser l'éducation critique aux médias à l'ère du numérique

\section{Présentation du modèle en littératie médiatique}

La définition du concept de littératie médiatique à laquelle nous nous référerons dans la suite de cet article (Fastrez 2010 ; Fastrez et De Smedt, 2012) se structure autour d'une double distinction: d'une part entre quatre formes d'activité médiatique ; d'autre part entre trois types d'objets médiatiques.

Concernant les activités, nous distinguons la lecture et l'écriture, centrées respectivement sur la réception et la production d'un seul objet médiatique, et la navigation et l'organisation, centrées respectivement sur la réception et la production d'une collection d'objets médiatiques entretenant des relations.

Concernant les objets, nous posons que tout objet (document, message, dispositif, appareil) médiatique peut être considéré tour à tour, comme un objet technique (fabriqué, ou qui fabrique), informationnel (qui représente) et social (qui met des personnes, des institutions et des communautés en relation).

Les objets médiatiques sont ainsi des objets techniques. Ce sont des artefacts: ils sont fabriqués par l'homme dans des matériaux plus ou moins complexes. Différents dispositifs techniques permettent de les produire, de les maintenir, de les acheminer où l'on veut qu'ils parviennent, ou de les rendre manifestes pour leurs récepteurs. Certains servent eux-mêmes à produire ou à diffuser d'autres objets médiatiques (un lecteur Blu-ray, un moteur de recherche, un téléphone cellulaire, un logiciel de traitement de texte sont autant d'objets médiatiques requérant une interaction technique).

Les objets médiatiques constituent également des objets informationnels. Ils sont conçus pour représenter des objets, réels ou fictifs, qui leur sont extérieurs, à travers l'usage de langages qui leur sont propres. Par la médiation de codes, de savoirs de nature encyclopédique, de schèmes et de types cognitifs et de multiples processus d'inférence, plus ou moins largement partagés entre plusieurs personnes, ils permettent d'évoquer un concept, une idée, une valeur ou un sentiment.

Enfin, les objets médiatiques constituent des objets sociaux, en ce qu'ils tissent des relations sociales entre les membres de 
la société. Ils renvoient aux acteurs (institutions et personnes) qui les produisent et les diffusent, aux intentions de ces acteurs, aux effets qu'ils produisent sur leurs publics, aux modèles culturels qu'ils véhiculent, aux principes liés à leur usage responsable et éthique.

Les compétences réunies sous l'appellation «littératie médiatique » sont donc celles rendant l'individu capable de lire, d'écrire, de naviguer et d'organiser les objets médiatiques en tant qu'objets techniques, informationnels et sociaux (Tableau 1). À travers ces quatre formes d'activités, l'individu compétent peut considérer les objets médiatiques avec lesquels il interagit (et les relations entre ceux-ci): du point de vue (informationnel) de ce que ces objets donnent à voir, des systèmes langagiers qu'ils utilisent et de la façon dont les propriétés formelles de ces langages rendent possibles différents processus de signification; du point de vue (technique) des procédés techniques sous-tendant leur production ou leur réception ; ou du point de vue (social) de leur contexte de production institutionnel, des intentions de leurs auteurs, des stéréotypes culturels qu'ils renforcent, etc.

Tableau 1. Les formes d'activités médiatiques

\begin{tabular}{|l|l|}
\hline Lire & $\begin{array}{l}\text { Décoder, comprendre, évaluer des objets } \\
\text { médiatiques }\end{array}$ \\
\hline Naviguer & $\begin{array}{l}\text { Chercher des objets médiatiques et explorer } \\
\text { des offres médiatiques }\end{array}$ \\
\hline Écrire & $\begin{array}{l}\text { Créer et diffuser ses propres productions } \\
\text { médiatiques }\end{array}$ \\
\hline Organiser & $\begin{array}{l}\text { Catégoriser des objets médiatiques à l'aide de } \\
\text { typologies ad hoc }\end{array}$ \\
\hline
\end{tabular}

\section{Penser ce modèle pour une éducation critique aux médias}

Cette présentation succincte pourrait mener à concevoir les trois dimensions (technique, informationnelle, sociale) précitées comme étant indépendantes. Dans la section qui suit, nous nous emploierons au contraire à mettre en exergue les relations 
Un modèle pour repenser l'éducation critique aux médias à l'ère du numérique

étroites qui lient ces trois dimensions et en quoi leur articulation est propre à une éducation critique aux médias à l'ère du numérique.

\section{1. Évaluation de l'information et esprit critique}

Les premières définitions de la littératie médiatique, formulées à l'heure de la structuration du champ de l'éducation aux médias, paraissent rétrospectivement essentiellement focalisées sur des questions informationnelles. Par exemple, dans son rapport sur la National Leadership Conference on Media Literacy, Aufderheide proposait la définition suivante, qui allait devenir la référence du champ : " the ability of a citizen to access, analyze, and produce information for specific outcomes" (Aufderheide et Firestone, 1993, p. 6, nous soulignons) $)^{3}$. Le récent rapprochement entre éducation aux médias et éducation à l'information au sein de l'UNESCO, sous l'appellation " éducation aux médias et à l'information " (Catts et Lau, 2008 ; Moeller, Ammu, Lau et Carbo, 2011), montre que l'essor du numérique a contribué à établir cette centration sur l'information. La recherche d'information sur Internet et la capacité à évaluer celle-ci et ses sources sont par conséquent devenues une dimension centrale des compétences médiatiques (Serres, 2012).

De façon générale, l'analyse et l'évaluation de l'information ou des autres contenus médiatiques sont par ailleurs les compétences les plus communément couplées à la notion d'esprit critique, ou de pensée critique, faisant de la littératie médiatique « une aptitude de pensée critique rendant les publics capables de développer des jugements indépendants à propos du contenu des médias " ${ }^{4}$ (Silverblatt, 2001, p. 2). À travers cette association entre évaluation et pensée critique, l'apparente centration de l'éducation aux médias sur des questions informationnelles se révèle en fait porter tout autant

\footnotetext{
${ }^{3}$ Nombreux sont les textes et les institutions qui ont depuis repris le triptyque " accéder, évaluer, produire » pour définir la littératie médiatique, sous des variantes remplaçant " information » par " média » ou " communications » (par exemple Buckingham, 2005 ; Livingstone et al., 2005, relayant la définition de l'OFCOM, Beekhus et Zaccheti 2009).

${ }^{4}$ Traduction libre de : " a critical thinking skill that enables audiences to develop independent judgments about media content ».
} 


\section{Pierre FASTREZ et Thibault PHILIPPETTE}

sur des questions sociales. En effet, ce que la recherche en éducation aux médias a le plus souvent désigné comme pensée critique consiste précisément à connecter l'information médiatique à ses contextes (sociaux) de production et de réception.

Dans le monde anglo-saxon, cette forme de contextualisation est travaillée depuis longtemps au départ d'une pédagogie de la déconstruction, structurée autour d'un nombre limité de concepts-clés ( $p$. ex. Jolls et Wilson, 2014), couplés à autant de questions-cadres au départ desquelles l'apprenant doit pouvoir s'interroger sur les médias qu'il rencontre. Si ce couplage concepts-questions a connu de nombreuses versions et itérations depuis les années 1980 (p. ex. Bazalgette, 1989), nous citerons ici, à titre d'exemple, la formulation récente qu'en proposent Funk, Kellner et Share (2016, pp.7-8, notre traduction) :

- Constructivisme social: qui sont toutes les personnes qui ont pu faire des choix contribuant à la création de ce texte?

- Langages et sémiotique: comment ce texte a-t-il été construit, diffusé et comment y a-t-on eu accès ?

- Publics et positionnements : Comment ce texte pourrait-il être compris différemment?

- Politique de la représentation: Quelles valeurs, points de vue et idéologies sont représentés ou manquants dans ce texte ou influencé par ce médium ?

- Production et institutions: Pourquoi ce texte a-t-il été créé et/ou partagé ?

- Justice sociale: qui ce texte avantage-t-il ou désavantage-t-il ?

Abordées au départ de ces six concepts-clés et de leurs questions associées, l'analyse et l'évaluation critique des médias ont donc bien pour objet de les envisager sous des perspectives multiples, mettant au jour les relations entre leurs contenus informationnels, les ressources sémiotiques qu'ils mobilisent, les personnes, les institutions et les communautés qu'ils relient. On voit comment cette contextualisation sociale de l'information renvoie aux questions de pouvoir, de représentations et d'idéologies centrales à la critical media literacy (cf. 1.1.2). 
Un modèle pour repenser l'éducation critique aux médias à l'ère du numérique

Si on l'interprète à la lumière des catégories définies par la matrice des compétences décrite plus haut, cette approche de la littératie médiatique apparaît centrée sur les compétences (en réception) à lire et à naviguer parmi les objets médiatiques d'un point de vue socio-informationnel. Dans cette perspective, la production (écrire et organiser ces objets) n'est souvent mobilisée que comme un moyen de développer les compétences analytiques : en apprenant à fabriquer du média, on suppose que les élèves apprendront à être critiques vis-à-vis des médias, et de nombreuses recherches se sont employées à vérifier cette hypothèse ( $p$. ex. Hobbs, Donnelly, Friesem et Moen, 2013 ; Phang et Shaefer, 2009).

On peut pourtant concevoir que l'individu éduqué aux médias mobilise ses aptitudes à la critique socio-informationnelle tant en situation de production que de réception et applique à ses propres productions médiatiques les mêmes interrogations que celles décrites plus haut, par exemple :

- À quel titre prends-je la parole dans l'espace public?

- Quelles sont mes intentions en produisant ce message ?

- Quels sont mes destinataires et comment mon message les qualifie-t-il ?

- Quelles valeurs ou quels points de vue sont présents dans mon message, et lesquels ai-je omis ?

Une autre particularité de cette approche traditionnelle de la littératie médiatique tient au peu de place qu'elle accorde à la troisième dimension des objets médiatiques figurant dans notre modèle matriciel: la technique. En effet, dans l'évolution historique des concepts-clés au départ desquels les éducateurs aux médias déconstruisent les objets médiatiques avec leurs élèves, l'examen des technologies sous-tendant la production des médias (Bazalgette, 1989 ; Wangermée, 1995) a laissé la place aux « techniques" utilisées pour attirer et convaincre le récepteur. Ces techniques sont conçues comme des procédés sémiotiques, et non comme des procédés technologiques de fabrication, de diffusion ou de réception.

Dans la section suivante, nous envisagerons la place de la technique dans la définition des compétences numériques. 


\section{Pierre FASTREZ et Thibault PHILIPPETTE}

\subsection{Des compétences techniques élémentaires et conformistes}

Aux côtés de la problématique de la littératie médiatique, celle des compétences numériques a rapidement émergé comme un enjeu sociétal majeur, notamment aux yeux du monde politique, qui y voit un facteur de lutte contre l'exclusion sociale. Les possibles rapprochements entre littératie médiatique et compétences numériques constituent également un risque : celui de voir la richesse du concept de littératie médiatique considérablement réduit.

Buckingham (2006) a bien résumé en quoi l'expression « compétences numériques » (ou digital skills) désignait trop souvent un ensemble très restreint d'aptitudes fonctionnelles :

Dans l'usage contemporain, la littératie numérique (ou informatique) paraît souvent être limitée à un ensemble minimal de savoir-faire qui rendront l'usager capable de fonctionner efficacement avec des outils logiciels, ou d'accomplir des tâches basiques de recherche d'information. II s'agit essentiellement d'une définition fonctionnelle : elle spécifie les capacités de base qui sont requises pour entreprendre des opérations particulières, mais elle ne va pas beaucoup plus $\operatorname{loin}^{5}$ (p. 265).

De ce point de vue, les compétences numériques apparaissent, au contraire de la littératie médiatique, définies essentiellement du point de vue de la technicité et borné à des capacités élémentaires. Cette affirmation doit cependant être nuancée, dans la mesure où il existe bien, dans la littérature, des modèles des compétences numériques dépassant les simples capacités opérationnelles décrites par Buckingham. Les travaux de van Deursen et van Dijk $(2005,2014)$ en constituent un bon exemple. Les deux auteurs distinguent six catégories de capacités ${ }^{6}$ numériques, ordonnées de façon

\footnotetext{
${ }^{5}$ Traduction libre de : «In contemporary usage, digital (or computer) literacy often appears to amount to a minimal set of skills that will enable the user to operate effectively with software tools, or in performing basic information retrieval tasks. This is essentially a functional definition: it specifies the basic skills that are required to undertake particular operations, but it does not go very far beyond this. "

6 Nous traduisons ici skill par capacité et non par compétence. La gradation proposée par van Deursen et van Dijk rend difficile la résolution de cette question de traduction, les premières catégories s'apparentant bien à des capacités (au sens d'aptitudes à reproduire des procédures apprises telles quelles) et les dernières, à des compétences (au sens d'aptitudes à choisir et à mobiliser de façon flexible, parmi un répertoire de capacités, de connaissances et d'attitudes, celles qui sont les
} 
Un modèle pour repenser l'éducation critique aux médias à l'ère du numérique

séquentielle et conditionnelle ${ }^{7}$ : les capacités opérationnelles (utiliser un ordinateur et l'Internet), formelles (portant sur les propriétés formelles des médias numériques), informationnelles (recherche, sélection et évaluation de l'information), de communication, de création de contenu, et stratégiques (usage des TIC à des fins de satisfaction d'objectifs personnels ou professionnels). Dans ce modèle, les capacités techniques, cantonnées aux niveaux opérationnel et formel, constituent donc le socle sur lequel les compétences de plus haut niveau (que nous qualifierons d'informationnelles et de sociales) se construisent.

Dans ce qui précède, la dimension technique des compétences est donc soit largement absente (en littératie médiatique), soit limitée à un niveau d'accomplissement élémentaire (dans les compétences numériques). Au-delà du constat que technique rime ici avec basique ${ }^{8}$, cette situation nous semble problématique en ce qu'elle laisse transparaître une conception des usages de la technologie fondée dans un conformisme a-critique (Collard et al., 2017). II s'agit en effet de borner les compétences à appréhender les objets médiatiques dans leur dimension technique aux capacités à en assurer l'opération correcte, afin de pouvoir fonctionner efficacement dans la vie quotidienne. Ceci reflète une conception du progrès technologique comme étant inéluctable et indépendant de l'usager, qui doit, pour s'adapter à son environnement technique, rester "à jour » dans sa capacité à bien l'utiliser, pour ne pas être marginalisé.

Afin de se dégager de cette vision réductrice, il nous semble utile de considérer les compétences se trouvant à l'articulation du technique et de l'informationnel, ou du social, qui délimitent un espace dépassant l'usage conformiste, où la critique et la créativité ont leur place.

Dans les deux sections suivantes, nous explorerons deux pistes envisageant comment, dans les contextes d'usage

plus appropriées à la résolution d'une tâche complexe et inédite pour l'usager).

7 Chaque catégorie constitue un prérequis nécessaire au développement de la suivante.

${ }^{8}$ II est pourtant aisé de concevoir que des compétences techniques avancées (en programmation, en manipulation du son et de l'image, en électronique...) fassent partie de la littératie médiatique des individus, même si celles-ci sont plus souvent maîtrisées par les professionnels des médias. 
propres aux médias numériques plaçant l'usager tant en position de contributeur que de récepteur, une compréhension et un usage avancés de la dimension technique des médias mettent celles-ci en regard de leurs dimensions respectivement informationnelle et sociale.

\subsection{Les objets techniques comme artefacts cognitifs}

La première de ces deux pistes s'ancre dans la théorie de la cognition distribuée (Hutchins, 1995), et plus spécifiquement dans l'une des trois formes de distribution de la cognition identifiée par Hutchins (2001) : celle impliquant la coordination de structures internes (mentales) et externes (matérielles). Dans ce cadre, les technologies de l'information et de la communication peuvent être conçues comme des artefacts cognitifs, des " outil[s] artificiel[s] conçu[s] pour conserver, exposer et traiter l'information dans le but de satisfaire une fonction représentationnelle »(Norman, 1993, p. 18).

Les artefacts cognitifs fonctionnent comme des technologies de l'intelligence (Lévy, 1990). Le système formé du couplage entre l'individu et l'artefact possède des aptitudes cognitives que l'individu seul ne possède pas. Les artefacts cognitifs remplissent leurs fonctions du fait que leur manipulation rend disponibles (c'est-à-dire directement perceptibles) des informations autrement inaccessibles, ou devant être calculées ou imaginées mentalement. En cela, l'usage de l'artefact pallie les limitations de notre système cognitif propre (c'est-à-dire non assisté), en soutenant une distribution sémiotique de la cognition, dans laquelle les représentations externes fournies par l'artefact, et leur production, leur transformation et leur perception, se substituent aux représentations internes de l'individu et aux opérations mentales présidant à leur traitement.

Nous voyons dans cette théorie une ressource importante pour l'examen de la littératie médiatique. En effet, au-delà du constat que l'être humain ne possède et ne déploie ses "pouvoirs cognitifs" que grâce à l'environnement qu'il a façonné, il paraît opportun de souligner en quoi l'exploitation de ressources environnementales sémiotiques à des fins cognitives repose sur des compétences spécifiques, qui sont à la croisée du technique et de l'informationnel. Jenkins et ses 
Un modèle pour repenser l'éducation critique aux médias à l'ère du numérique

collaborateurs (2006) font ainsi figurer la cognition distribuée, décrite comme "l'aptitude à interagir de façon signifiante avec les outils qui étendent nos capacités mentales ", parmi leur liste des onze nouvelles formes de littératie médiatique. Sans prétention d'exhaustivité, nous listons ci-dessous quelquesunes de ces compétences :

- percevoir les affordances des technologies de communication, qu'elles soient pragmatiques (qu'est-ce que l'outil me permet de faire ?) ou épistémiques (qu'estce qu'il me permet de connaître ?) ;

- concevoir en quoi une technologie médiatique change l'activité qu'elle soutient et les aptitudes nécessaires à son accomplissement ;

- choisir une technologie médiatique en regard des exigences de l'activité qu'elle soutient, en fonction de ses affordances (que celles-ci correspondent ou non aux prescriptions de son concepteur [Bardini, 1996]) et contraintes, de sorte à adapter l'environnement de l'activité pour qu'il soit plus cognitivement accueillant (cognitively congenial [Kirsh, 1996]) ;

- considérer la représentation comme distincte de la chose représentée et utiliser les propriétés formelles de la représentation pour raisonner sur la chose représentée (Hollan, Hutchins et Kirsh, 2000) ;

- considérer l'interface d'un artefact comme un système sémiotique assurant la traduction entre ses représentations de surface et ses représentations internes (Norman, 1993).

\subsection{Identités numériques, algorithmie et sociomatique}

La seconde piste, articulant technique et social, concerne l'avènement du web social, des réseaux socionumériques et des technologies de partage et de collaboration. Dans ce contexte, chacun dispose désormais d'un nombre croissant d'opportunités de se présenter au monde et d'établir et d'entretenir des contacts et des relations diverses par des voies médiatisées. Les technologies de communication donnent forme à ces nouveaux modes d'interaction sociale ${ }^{9}$.

\footnotetext{
${ }^{9}$ Notre propos n'est ni d'affirmer que ceci est vrai seulement pour le web social -
} 


\section{Pierre FASTREZ et Thibault PHILIPPETTE}

L'appréhension des relations entre technique et socialité appelle des compétences spécifiques. Nous les développerons ici à deux égards.

Premièrement, il s'agit d'envisager comment les sites, les applications et les services médiant nos relations en ligne imposent un format à celles-ci. C'est le cas pour les formes d'expression des identités numériques. Aux réseaux socionumériques correspond une sémiotique spécifique des signes de soi en ligne, sédimentée sous la métaphore du profil (Georges, 2010). Cette sémiotique est largement définie par l'infrastructure technique qui la sous-tend, de sorte que les façons de se donner à voir aux yeux des autres sont cadrées par des formats standardisés déterminés par l'industrie du web. Une conception naïve de ce phénomène dénoncerait la façon dont la technique déforme ce que nous sommes. II s'agit plutôt de «penser les identités comme des constructions technosémiotiques matérialisées à l'écran et non pas comme des essences qui préexisteraient » (Gomez-Meija, 2016, p. 22).

De même que nos identités numériques, l'infrastructure technique produite par les industries du web régule et arbitre nos relations sociales: les comptes d'utilisateurs définissent des rôles, les rôles définissent des accès. Désormais, dans un nombre croissant de contextes quotidiens personnels ou professionnels, la réponse aux questions «qui peut parler à qui ?», "qui peut voir ou lire quoi ? " ou "qui peut faire quoi ? " est déterminée par une procédure, un filtre, un verrou électronique préalablement paramétrés.

Si l'informatique est définie comme le traitement automatique de l'information, nous définissons la sociomatique comme le traitement automatique des interactions sociales, tel que nous venons de le décrire brièvement. La compréhension de la sociomatique correspond selon nous à un ensemble de compétences permettant à l'usager des TIC d'évaluer de façon critique les conséquences des choix techniques sur la régulation des interactions sociales et d'adopter un comportement d'usage tenant compte de cette évaluation.

toute forme de relation médiatisée est effectivement influencée par les modalités techniques de sa médiatisation - ni de prétendre que la technique détermine à elle seule les formes d'interaction sociale médiatisée - la sociologie des usages a depuis longtemps déconstruit l'argument d'un déterminisme technique (Millerand, Proulx et Rueff, 2010). 
Un modèle pour repenser l'éducation critique aux médias à l'ère du numérique

Deuxièmement, une composante complémentaire de la sociomatique est liée au rôle croissant que joue le traitement algorithmique dans nos interactions médiatisées. Plus encore que les signes identitaires auxquels les supports techniques donnent forme et existence, la matière première dont se nourrissent désormais les médias numériques est faite des collections pléthoriques de traces (Merzeau, 2009) que leurs usagers laissent derrière eux de façon inévitable ${ }^{10}$ en cliquant, en " likant », en achetant, en se géolocalisant, etc.

Cardon (2015) a bien décrit comment les algorithmes de traitement automatique de l'information (et, ce faisant, des interactions sociales), sur lesquels repose un nombre croissant de dispositifs médiatiques, travaillent sur la base des signes publiés en ligne et des traces d'usage. L'auteur met au jour les différentes représentations de la société que quatre familles d'algorithmes produisent, selon que leur principe de fonctionnement soit fondé sur :

- la popularité : en comptant le nombre de vues, on renvoie à l'usager le monde tel que le plus grand nombre le voit ;

- l'autorité : en comptant le nombre de liens pointant vers une ressource, on lui donne à voir un monde validé par des sources reconnues ;

- la réputation: en comptant le nombre de likes, on lui montre le monde tel que ses amis l'aiment ;

- la prédiction : en corrélant ses traces à celles d'autres usagers qui lui ressemblent, on lui présente un monde conforme à ses actions passées, prédisant ses actions futures.

Selon qu'un algorithme privilégie l'un de ces principes, les informations présentées à l'usager dépendront de la quantification des internautes-lecteurs (popularité), des producteurs d'information (autorité), de ses amis (réputation), ou de soi-même (prédiction), chaque algorithme renvoyant la responsabilité de la façon dont le monde apparaît sur écran à l'une de ces instances. On voit ici que la question des représentations produites par les médias, chère aux tenants de

\footnotetext{
${ }^{10}$ À l'heure du numérique, " on ne peut plus ne pas laisser de traces », dit Merzeau (2009), paraphrasant le premier axiome de la communication formulé par l'école de Palo Alto (Watzlawick, Helmick Beavin et Jackson, 1972).
} 


\section{Pierre FASTREZ et Thibault PHILIPPETTE}

la critical media literacy, trouve un nouveau terrain d'application, d'autant plus crucial que les choix politiques et moraux opérés par les concepteurs des algorithmes, qui président à ce que ceux-ci produisent comme représentations, sont largement invisibles pour le grand public.

Qui plus est, dans le cas des algorithmes prédictifs, cette question des représentations se pose désormais en relation directe avec les caractéristiques de l'usager, ses traces d'usages déterminant la façon dont le système prédira ses préférences (affinitaires, professionnelles, religieuses, politiques, culturelles...) et lui renverra un monde informationnel confirmant celles-ci.

On le voit, les compétences de compréhension de la sociomatique apparaissent ici essentielles, en ce qu'elles peuvent aider l'usager à s'interroger sur les propositions du système et à explorer les mondes numériques en dehors de celles-ci, en cultivant son ouverture à la sérendipité.

\section{Conclusion}

Si une éducation critique aux médias a pour objectif de développer une pensée critique et réflexive vis-à-vis des médias, elle doit pour ce faire tenir compte des caractéristiques problématiques de la situation médiatique, se fonder sur des pédagogies permettant d'intégrer les points de vue de tous les acteurs impliqués, mais également faire acquérir les compétences nécessaires pour agir en vue d'améliorer les conditions de cet environnement médiatique.

À travers la présentation de notre matrice de référence en littératie médiatique, nous avons voulu mettre en exergue un cadre permettant de penser ces compétences à acquérir, définies par des combinatoires possibles des dimensions identifiées. Une éducation critique doit selon nous articuler explicitement les dimensions techniques, informationnelles et sociales des objets médiatiques, tant dans les activités de réception (lecture et navigation) que de production (écriture et organisation).

Nous avons illustré le fait qu'une littératie médiatique critique à l'ère du numérique concerne aussi bien l'analyse et l'évaluation critique des objets médiatiques (combinant 
Un modèle pour repenser l'éducation critique aux médias à l'ère du numérique

informationnel et social), la technique et l'informatique pensés et conçus comme des artefacts cognitifs (combinant informationnel et technique) et les configurations sociales engendrées par les outils et les algorithmes que nous avons qualifiés de "sociomatiques" (combinant social et technique). Ces combinatoires constituent une manière simple de montrer qu'il faut penser cette matrice en articulant les dimensions plutôt qu'en les séparant. Dans la plupart des situations médiatiques, les trois dimensions jouent un rôle conjoint pour pouvoir comprendre, analyser et concevoir des médias de manière critique.

Le champ de l'éducation aux médias a une longue tradition de conversion des théories et des résultats de la recherche sur les médias en contenus d'enseignement et en référentiels de compétences constituant les objectifs des processus éducatifs qu'elle met en œuvre. Les théories sémiotiques et narratologiques, les études de genre, les études culturelles, pour ne citer qu'elles, ont toutes abondamment irrigué l'éducation aux médias. II nous semble aujourd'hui nécessaire que les champs de recherche esquissés dans la dernière partie de cet article (cognition distribuée et sociomatique) viennent grossir les rangs des cadres de pensée utilisés en éducation aux médias pour couvrir tous les aspects d'une conception et d'une évaluation critiques des médias à l'ère numérique.

\section{Références}

ALVERMANN D. E. et M. C. HAGOOD, 2000, «Critical Media Literacy: Research, Theory, and Practice in "New Times" ", The Journal of Educational Research, vol. 93, n' 3, pp. 193205.

ANDERSON J.A., 1988, "Examen de quelques concepts éclairant la position de l'éducateur aux médias ", dans Rencontre de la recherche et de l'éducation: actes du Symposium, 27-30 juin 1988, Lausanne (Suisse), Centre d'initiation aux communications de masse, pp. 11-23.

ANDUREAU J.-P., 2003, "Assujettissement et subjectivation : réflexions sur l'usage de Foucault en éducation », Revue française de pédagogie, vol. 143, n 1, pp. 17-29. 


\section{Pierre FASTREZ et Thibault PHILIPPETTE}

AUFDERHEIDE P. et C. M. FIRESTONE, 1993, Media Literacy: a Report of the National Leadership Conference on Media Literacy, Washington, Aspen Institute, Communications and Society Program.

BARDINI T., 1996, "Changement et réseaux sociotechniques: de l'inscription à l'affordance ", Réseaux, vol. 14, n 76, pp. 125-155.

BAZALGETTE C., 1992, "Key Aspects of Media Education », dans M. ALVARADO et O. BOYD-BARRETT (dir.), Media Education: An Introduction, Londres, British Film Institute \& Open University Press, pp. 199-219.

BAZALGETTE C. (dir.), 1989, Primary Media Education: A curriculum statement, Londres, British Film Institute.

BROTCORNE P., L. DAMHUIS, V. LAURENT, G. VALENDUC et P. VENDRAMIN, 2010, Diversité et vulnérabilité dans les usages des TIC. La fracture numérique au second degré, Gand, Academia Press.

BUCKINGHAM D., 1998, "Media education in the UK: Moving beyond protectionism », Journal of Communication, vol. 48, $\mathrm{n}^{\circ} 1$, pp. 33-43.

BUCKINGHAM D., 2006, "Defining digital literacy - What do young people need to know about digital media? ", Nordic Journal of Digital Literacy, vol. 1, n 4, pp. 263-276.

BUCKINGHAM D., 2013, Media Education: Literacy, Learning and Contemporary Culture, New York, John Wiley \& Sons.

CARDON D., 2015, A quoi rêvent les algorithmes: nos vies à l'heure des big data, Paris, Le Seuil.

CATTS R. et J. LAU, 2008, Towards information literacy indicators, Paris, UNESCO.

COLLARD, A.-S., T.DE SMEDT, M. DUFRASNE, P. FASTREZ, V. LIGURGO, G. PATRIARCHE et T. PHILIPPETTE, 2017, "Digital media literacy in the workplace : A model combining compliance and inventivity ", Italian Journal of Sociology of Education, vol. 9, $\mathrm{n}^{\circ} 1$, pp. 122-154. 
Un modèle pour repenser l'éducation critique aux médias à l'ère du numérique

DE MUNCK J., 2011, "Les trois dimensions de la sociologie critique », SociologieS, <http://sociologies.revues.org/3576>, dernière consultation le 15 décembre 2016.

DURHAM M., et D. KELLNER (dir), 2012, Media and Cultural Studies: Keyworks ( $2^{\mathrm{e}}$ edition), Malden, Wiley-Blackwell.

FASTREZ P., 2010, "Quelles compétences le concept de littératie médiatique englobe-t-il ? Une proposition de définition matricielle », Recherches en Communication, vol. 33, pp. 35-52.

FASTREZ P. et T. DE SMEDT, 2012, «Une description matricielle des compétences en littératie médiatique », dans Lebrun-Brossard M., N. Lacelle et J.-F. Boutin (dir), La littératie médiatique multimodale. De nouvelles approches en lecture-écriture à l'école et hors de l'école, Québec, Presses de l'Université du Québec, pp. 45-60.

FEUERSTEIN M., 1999, " Media literacy in support of critical thinking ", Journal of Educational Media, vol. 24, $\mathrm{n}^{\circ} 1$, pp. 43-54.

FUNK S., D. KELLNER et J. SHARE, 2016, "Critical Media Literacy as Transformative Pedagogy ", dans M. N. Yildiz et J. Keengwe (dir.), Handbook of Research on Media Literacy in the Digital Age, Hershey, IGI Global, pp. 1-30.

GAINER J., 2010, "Critical Media Literacy in Middle School: Exploring the Politics of Representation », Journal of Adolescent \& Adult Literacy, vol. 53, n5 , pp. 364-373

GEORGES F., 2010, Identités virtuelles. Les profils utilisateur du web 2.0, Paris, Questions Théoriques.

GIROUX H., 1983, "Theories of Reproduction and Resistance in the New Sociology of Education: A Critical Analysis", Harvard Educational Review, vol. 53, n 3, pp. 257-293.

GOMEZ-MEJIA G., 2016, Les Fabriques de Soi ? Identité et Industrie sur le web, MkF Éditions.

GUR-ZE'EV I., 2000, "Critical Education in Cyberspace? », Educational Philosophy \& Theory, vol. 32, n² 2, pp. 209-231.

HOBBS R., K. DONNELLY, J. FRIESEM et M. MOEN, 2013, " Learning to engage: How positive attitudes about the news, media literacy, and video production contribute to adolescent 


\section{Pierre FASTREZ et Thibault PHILIPPETTE}

civic engagement », Educational Media International, vol. 50, $\mathrm{n}^{\circ} 4$, pp. 231-246.

HOBBS R., 1998, «The seven great debates in the media literacy movement », Journal of Communication, vol. 48, $\mathrm{n}^{\circ} 1$, pp. 16-32.

HOLLAN J.D., E. L. HUTCHINS et D. KIRSH, 2000, « Distributed cognition: Toward a new foundation for humancomputer interaction research », ACM Transactions on Computer-Human Interaction, vol. 7, pp. 174-196.

HUTCHINS E. L., 1995, Cognition in the Wild, Cambridge, The MIT Press.

HUTCHINS E. L., 2001, «Distributed cognition », dans N. J. Smelser et P. B. Baltes (dir.), International Encyclopedia of the Social and Behavioral Sciences, Amsterdam, Elsevier Press, pp. 2068-2072.

JENKINS H., R. Purushotma, K. Clinton, M. Weigel et A. J. Robison, 2006, Confronting the Challenges of Participatory Culture: Media Education for the $21^{\text {st }}$ Century (White paper), Chicago, The John D. and Catherine T. MacArthur Foundation.

JOLLS T. et C. WILSON, 2014, « The Core Concepts: Fundamental to Media Literacy Yesterday, Today and Tomorrow », Journal of Media Literacy Education, vol. 6, $\mathrm{n}^{\circ} 2$, pp. 68-78.

KELLNER D. et J. SHARE, 2005, «Toward Critical Media Literacy: Core concepts, debates, organizations, and policy ", Discourse: Studies in the cultural politics of education, vol. 26, $\mathrm{n}^{\circ} 3$, pp. 369-386.

KELLNER D. et J. SHARE, 2007, "Critical media literacy, democracy, and the reconstruction of education ", dans D. MACEDO et S. STEINBERG (dir.), Media literacy: $A$ reader, New York, Peter Lang Publishing, pp. 3-23.

KIRSH D., 1996, «Adapting the Environment Instead of Oneself », Adaptive Behavior, vol. 4, n³-4, pp. 415-452.

KLINE K., 2016, "Jean Baudrillard and the Limits of Critical Media Literacy », Educational Theory, vol. 66, n 5, pp. 641656. 
Un modèle pour repenser l'éducation critique aux médias à l'ère du numérique

LÉVY P., 1990, Les technologies de l'intelligence. L'avenir de la pensée à l'ère informatique, Paris, La Découverte.

LIVINGSTONE S., 2003, "The changing nature and uses of media literacy », Media@LSE Electronic Working Papers, $\mathrm{n}^{\circ} 4,<$ http://eprints.Ise.ac.uk/13476/>, dernière consultation le 15 décembre 2016.

MASTERMAN L., 1990, Teaching the Media (Reprint edition), New York, Routledge.

MERZEAU L., 2009, "Du signe à la trace : l'information sur mesure », Hermès, La Revue, vol. 53, pp. 21-29.

MILLERAND F., S. PROULX et J. RUEFF (dir.), 2010, Web social : mutation de la communication, Québec, Presses de I'Université du Québec.

MOELLER S., J. AMMU, J. LAU et T. CARBO, 2011, Towards Media and Information Literacy Indicators. Background Document of the UNESCO Expert Meeting (Bangkok, Thailand, 4 - 6 November 2010), Paris, UNESCO.

NORMAN D. A., 1993, "Les artefacts cognitifs", Raisons Pratiques, vol. 4, pp. 15-34.

O'BYRNE W., 2012, Facilitating Critical Evaluation Skills through Content Creation: Empowering Adolescents as Readers and Writers of Online Information (thèse de doctorat), University of Connecticut.

PAPOLA-ELLIS A. et T. EBERLY, 2015, «Critical Literacy: Going Beyond the Demands of Common Core », Illinois Reading Council Journal, vol. 43, n² 2, pp. 2-15.

PAUL R., 2012, " Dialogical Thinking: Critical Thinking Thought Essential to the Acquisition of Rational Knowledge and Passions ", Critical Thinking: What Every Person Needs To Survive in a Rapidly Changing World, Tomales, Foundation for Critical Thinking, pp. 268-287.

PHANG A. et D. J. SCHAEFER, 2009, «Is Ignorance Bliss? Assessing Singaporean Media Literacy Awareness in the Era of Globalization ", Journalism \& Mass Communication Educator, vol. 64, $\mathrm{n}^{\circ}$ 2, pp. 156-172.

PIETTE J., 1996, Education aux médias et fonction critique, Paris, L'Harmattan. 


\section{Pierre FASTREZ et Thibault PHILIPPETTE}

SCHARRER E., 2005, "Sixth Graders Take on Television: Media Literacy and Critical Attitudes of Television Violence ", Communication Research Reports, vol. 22, $\mathrm{n}^{\circ} 4$, pp. 325333.

SERRES A., 2012, Dans le labyrinthe. Évaluer l'information sur internet, Caen, C\&F Éditions.

SILVERBLATT A., 2001, Media Literacy: Keys to Interpreting Media Messages, Second Edition, Santa Barbara, Praeger Publishers.

SMITH L., 2013, "Towards a model of critical information literacy instruction for the development of political agency ", Journal of Information Literacy, vol. 7, $\mathrm{n}^{\circ}$ 2, pp. 15-32.

TINSON C. et C. McBRIDE, 2013, "Introduction to Special Issue: Hip Hop, Critical Pedagogy, and Radical Education in a Time of Crisis ", Radical Teacher, n ${ }^{\circ}$ 7, pp. 1-9.

TISDELL E., 2008, «Critical Media Literacy and Transformative Learning Drawing on Pop Culture and Entertainment Media in Teaching for Diversity in Adult Higher Education », Journal of Transformative Education, vol. 6, $\mathrm{n}^{\circ}$ 1, pp. 48-67.

UNESCO, 1982, The Grünwald Declaration on Media Education, <http://www.unesco.org/education/pdf/MEDIA_F.PDF>, dernière consultation le 16 août 2017.

VAN DIJK J., 2005, The Deepening Divide. Inequality in the Information Society, Londres, Sage Publications.

VAN DIJK J. A. G. M. et A. J. A. M. VAN DEURSEN, 2014, Digital Skills: Unlocking the Information Society, New York, Palgrave Macmillan.

WANGERMÉE R., 1995, L'éducation à l'audiovisuel et aux médias. Rapport du Conseil de l'éducation aux médias, Bruxelles, Communauté française de Belgique: Conseil de l'Education aux Médias.

WATZLAWICK P., J. HELMICK BEAVIN et D. D. JACKSON, 1972, Une logique de la communication, Paris, Le Seuil. 